\title{
Investigation of enterobacter aerogenes effects on heavy oil from biological degradation aspects by GC*GC technique
}

\begin{abstract}
The main cause of oil pollution of soil is the emergency situations within oil production, transportation and processing in the boundaries of industrial sites of chemical and petrochemical industries. Oil pollution leads to the deterioration of the agrophysics soil characteristics, namely to the dysfunction of the water, air, thermal, oxidationreduction and nutrient regimens. Microbial technologies are becoming accepted worldwide as cost-effective and environmentally friendly approaches to improve oil production. One of the methods to purify oil residue is bioremediation, in this way we Investigate Enterobacter Aerogenes effects on heavy oil by SARA, FT-IR, GC and GCxGC methods. The material required for the growth of Enterobacter Aerogenes is Carbon and Phosphate. On the one hand, this bacterium takes these nutrients from carbohydrates in our body. In addition, the crude oil is full of Hydrocarbon structures. So, the Enterobacter Aerogenes is injected with Thioglycollate broth to crude oil which was distillated and the samples were put for a month in an incubator for 30 days. The results this research illustrate this bacterium has been able to survive in oil by isolating straight-run carbons with short and weak bounds from asphaltene and resin structures, as well as utilizing sulfur, nitrogen and oxygen in the oil. Also, it is found out this bacterium has had a positive effect on making light oil from heavy crude oil. It should be noted this bacterium releases $\mathrm{CH} 4$, which can be used again if the gas is collected.
\end{abstract}

Keywords: GCxGC, FT-IR, entrobacter aerogenes, heavy oil, bioremediation
Volume 4 Issue 2 - 2019

\author{
Arash Rasti,' Mahmud Memariani,' \\ Mohammad Ali Riahi \\ 'Department of petroleum, Science and Research branch, \\ Islamic Azad University, Iran \\ ${ }^{2}$ Department of Solid Earth Physics, University of Tehran, Iran
}

Correspondence: Mohammad Ali Riahi, Department of Solid Earth Physics, University of Tehran, Tehran I.R. Iran, Emailmariahi@ut.ac.ir

Received: February 27, 2018 | Published: April 04, 2019

\section{Introduction}

These days, many methods have been employed to eliminate oil residue contaminants including use of natural and synthetic adsorbents, mechanical methods, catalytic failure... and etc. Basically, the use of environmentally friendly technologies is one of the relatively new approaches in various industries. One of the most particularly and highly regarded methods is the use of oil bacteria in oil industry. The presence of biological species in oil-rich regions indicates the ability of these microorganisms in surviving in crude oil. Uses of biological methods are practically economical and natural. One of the applications of biotechnology is the use of bacteria in various industries, including the oil industry. Microorganisms are able to use petroleum for growth as a source of carbon and power. Biotechnology is a method which by direct or indirect use of microorganism or changing the genetic structure of bacteria serves human beings. The removal of sulfur from fuels, clearing of sea levels from oil spills, removing oil residue, purifying industrial waste are considered as important achievements in biotechnology. ${ }^{1}$ In the various sectors of the oil industry leakage and the spread of oil pollution, including the field of oil production, refineries and transportation lines due to the ineffectiveness of processes and accidents, is inevitable. In addition, these pollutants are injurious to human health and environment. Moreover, there are expensive to be cleared. Bioremediation is a method that uses the ability of living organisms to increase the rate of destruction of pollutants. These bacteria are found in a variety of sources, including water, sewage, Soil contaminated with oil, petroleum residues... and by using various mechanisms including producing gases-like hydrogen, nitrogen, methane and carbon dioxide that reduce oil viscosity and improve the characteristics of oil flow-they remove pollutants. ${ }^{2-4}$ in this paper, we study the effect of Enterobacter Aerogenes on heavy oil. After growing these bacteria in the thioglycolate broth, heavy oil is added and the whole medium is put in incubator for 30days then, SARA, FT-IR, GC and GCxGC techniques are employed to evaluate this bacterium. E. Aerogenes is a gram-negative bacterium which can cause digestive disease. This bacterium belongs to the family Enterobacteriaceae. E. Aerogenes can be found in gastrointestinal tract. Furthermore, it can cause UTI (Urinary Tract Infections). ${ }^{5-7}$ This explanation shows up this bacterium typically cause no harm to healthy individual. The material required for growth are Carbon and Phosphate also this bacterium take this nutrient from carbohydrates in our body. On the other hand, the crude oil is full of Hydrocarbon structures and this bacterium need these nutrients for growth then inject bacteria to crude oil to analyze the effects of bacteria on heavy oil which would it separate carbon from organic structures instead of Hydrocarbon structures in our body or not. Saturate, Aromatic, Resin and Asphaltene (SARA) is an analysis method that separation crude oil components according to their polarizability and polarity. The saturate fraction consists of nonpolar hydrocarbon/material including straight-run, branched, and cyclic saturated hydrocarbons (paraffins). Aromatics, which contain one or more aromatic rings, are slightly more polarizable. The remaining two fractions, resins and asphaltenes, have polar substituents. The distinction between the two is that asphaltenes are insoluble in an excess of heptane (or pentane) whereas resins are miscible with heptane (or pentane).$^{8}$ Chromatography encompasses a series of techniques that have in common the separation of components of a mixture by a series of equilibrium operations that result in separation of the entities as a result of their partitioning (differential sorption) between two different phases, one stationary with a large surface and the other a moving phase in contact with the first. ${ }^{9}$ Comprehensive gas chromatography, namely $\mathrm{GCxGC}$, is the last destination reached 
by separation science. "It falls into the category of multidimensional techniques, mainly due to the association of two different mechanisms of separation. Compared to the more known MDGC (Multidimensional Gas Chromatography), GCxGC is more "comprehensive" toward the separation process involving sample analytes". ${ }^{10}$

\section{Sampling strategies; methodology and tools}

First $100 \mathrm{ml} /$ lit of crude oil from the well No. 113 of Gachsaran field was distilled for two hours to obtain heavy oil. Then, $15 \mathrm{mil} /$ lit heavy oil plus $15 \mathrm{mil} /$ lit of thioglycollate broth were added and mixed with a shaker for 2 hours. Finally, 150 Lambda $(\lambda)$ enterobacter aerogenes was added and mixed for 20 minutes, and then, they were put in an incubator for 30 days. SARA test results after 30days before and after the treatment regarding the bacterial effect on crude oil are:

i. This chart illustrates that the bacterium effected on all of the part of petroleum, and the saturation result as well as the asphaltene part decreased by $6 \%$ and $2 \%$, respectively. On the other hand, the aromatic part and resin increased by $6 \%$ and $2 \%$, respectively.

ii. Evidences show that this bacterium used straight-run alkane compounds instead of the power source. Also, it has effects on the resin section to release the heterocyclic carbon materials on the aromatic section.

\section{Comparison the results of bacterial function on the samples with FT-IR technique}

FT-IR analysis shows what molecules with what structure are present in a sample. ${ }^{11}$ Two types of infrared spectrometers are in common use in the organic laboratory: dispersive and Fourier transform (FT) instruments. Both of these types of instruments provide spectra of compounds in the common range of 4000 to $400 \mathrm{~cm}-1$. Although the two provide nearly identical spectra for a given compound, FTIR infrared spectrometers provide the infrared spectrum much more rapidly than the dispersive instruments. ${ }^{12}$ At the bottom, you can see the FT-IR results of samples before and after treatment. These charts illustrate the saturate part of the hydroxy- group and amine group with the small bound carbons deleted. Also, the long chain groups such as $\mathrm{OH}$ and $\mathrm{NH} 2$ increase slowly. Also, the pick number 1030 show that the oxygen - sulfur from sulfoxide group has been deleted. On the other hand, after treating the pick number 2723, making hydrogen - carbon with strong structure from aldehyde hydrogen group with weak bounds was considered. These changes represent the alcohol functional oxidation to aldehyde (Figure 1). The aromatic charts show the slowly deleting carbonyl group, which the picks number 1254 and 1024 confirmed it (Figure 2). Biodegradation occurred in Resin part, and then the smaller molecule with $\mathrm{OH}$ and $\mathrm{NH}$ functional groups Isolated from the asphaltene complex. The achievement of these changes is to increase the concentration of alcohol, amine and phenol (Figure 3). Finally, the asphaltene charts primarily show that hydrogen and nitrogen structures are deleted ${ }^{13}$ and also the peaks less than 1375 are destroyed too. These peaks include alcohol and aromatic compound with hydrogen structure. On the other hand, the tensile strength of alcohol and phenol groups increases, also the smaller alkane chain compound is isolated from the asphaltene part $(\mathrm{CH}$ and $\mathrm{CH} 2)$. These changes indicate the effects of bacteria on the biological degradation of the crude oil sample (Figure 4).

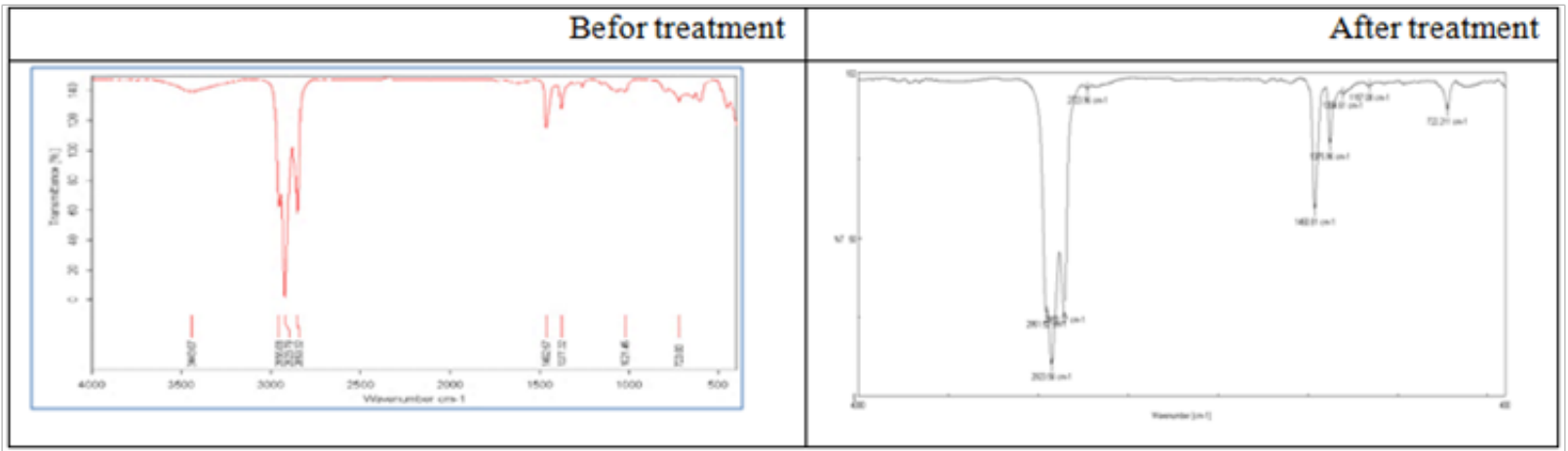

Figure I FT-IT result for saturate parts.

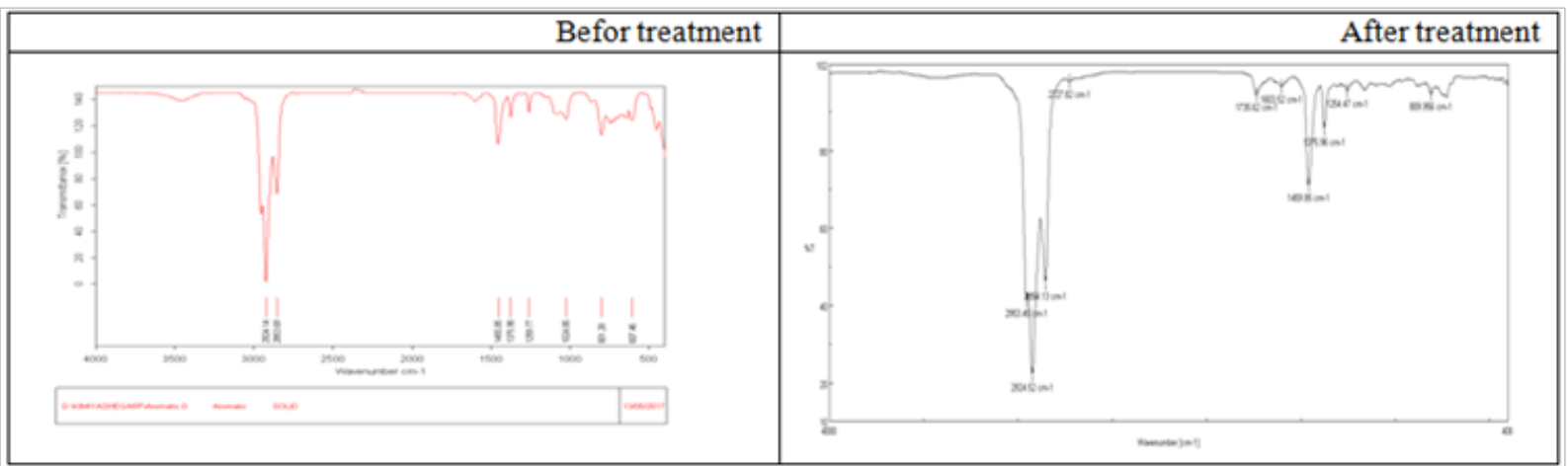

Figure 2 FT-IT result for aromatic parts. 


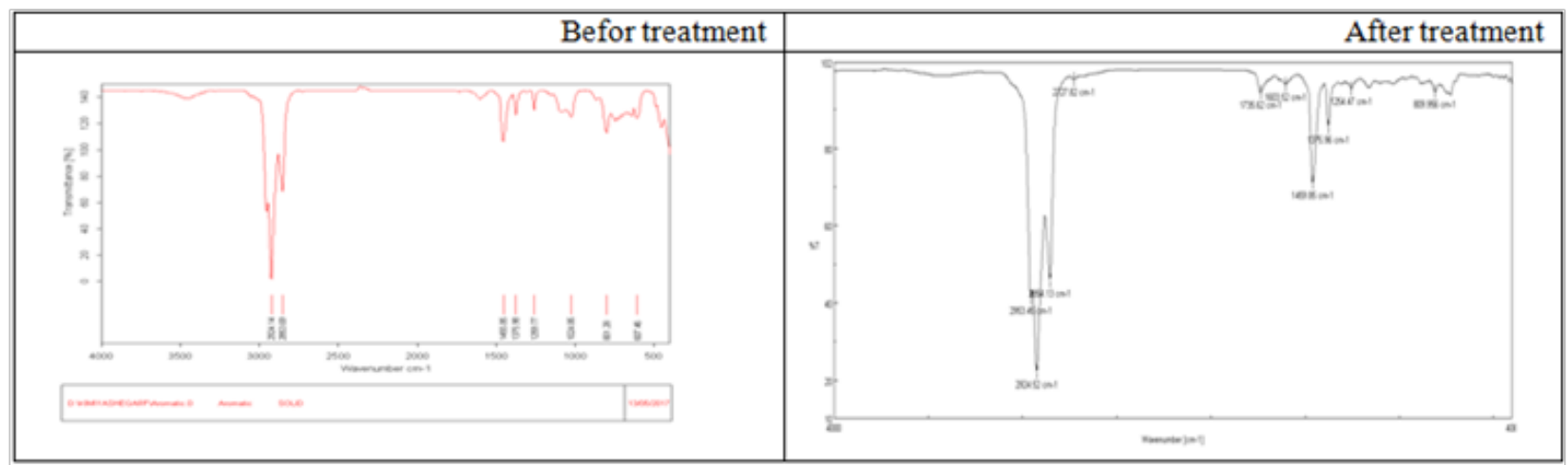

Figure 3 FT-IT result for resin parts.

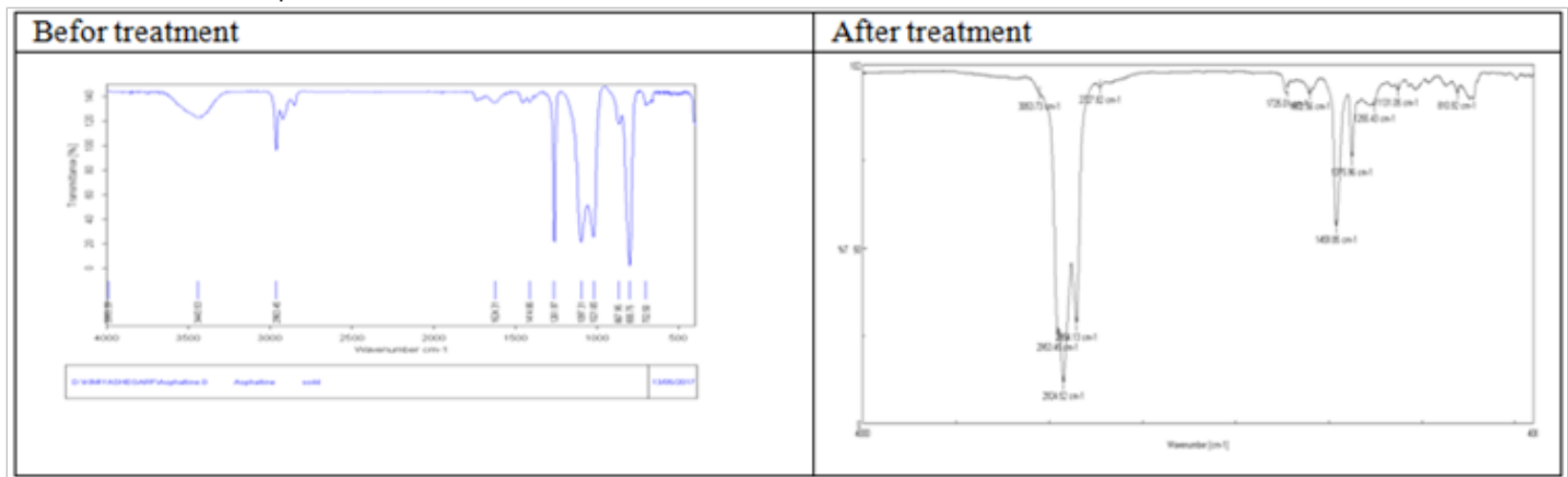

Figure 4 FT-IT result for resin parts.

\section{Gas chromatography interpretation}

To evaluate the bacterial function on the crude oil samples, the saturation parts before and after the treatment are evaluated by the gas chromatography. Since crude oil is a good source of nutrition for bacteria, comparison of the chromatograms obtained from the sample (before and after the treatment) can provide proper information on the bacterial functions in the crude oil sample. The spectrum obtained from the gas chromatography analysis is shown in Figure 5. According to the spectrum, the distribution of normal hydrocarbon starts from $\mathrm{C} 15$ to $\mathrm{C} 35$ in the spectrum field. On the other hand, the reason for the lack of light hydrocarbons (from carbon 8 to 14) is mainly due to the distillation of the crude oil. The aforementioned samples are analysed by a two-dimensional chromatography (GCxGC). The saturated part was analyzed by the gas chromatography after applying bacterial effects on the crude oil sample. ${ }^{14}$ The spectrum obtained from this test is shown in Figure 5. Evidence suggests that the distribution of the normal alkane components is different from the sample, before the treatment. The emergence of light hydrocarbons in the spectrum field shows that the effect of bacteria on increasing the fluidity of the crude oil sample was due to breaking the alkane chain connected to the polar sections of the sample, and their separation from resin and asphaltene complexes. The reduction of the of saturated hydrocarbon components concentration, especially alkanes higher than C26 in the field of spectrum shows the bacterial effect on the saturated section that uses the saturate compound instead of the power source. ${ }^{15}$ According to the above two illustrations, we can identify carbon No.16 to 34 in the first crude oil sample without bacteria; the crude oil is distilled and then the lighter hydrocarbon is deleted. The picture on the right shows the sample of crude oil affected by bacteria that leads to producing carbon NO.11 with high peaks, and eventually the peaks are terminated by carbon No. 29 and 30 . The two graphs clearly show the breaking of heavy hydrocarbon structures around the peak 17 to 31 , and eventually the formation of lighter carbon, ${ }^{16}$ as carbon numbers 11 to 16 can be seen. Carbons with a higher carbon number, have a longer alkaline chain with more hydrogen than hydrocarbon with smaller chain. Therefore, combining the results of IR and GC shows that this bacterium uses carbon NO.17 to 34 instead of the power source in the saturate part. Moreover, this bacterium uses the hydrocarbon in the aromatic part for producing lighter hydrocarbon, which includes hydrogen, and destroyed the alcohol structure in asphaltene part to use the hydrogen, nitrogen and oxygen as a power source. ${ }^{17}$

\section{Comprehensive two-dimensional gas chromatography interpretation}

Chemical analyses of the samples were evaluated with twodimensional chromatography technique before and after the treatment. The two-dimensional spectra obtained from this method are shown in Figures 6 and 7. Figure 6- Comparison of conventional and twodimensional GC spectra obtained from the $\mathrm{GC} \times \mathrm{GC}$ technique on saturation part from crude oil, before treatment (above spectrum A), ${ }^{18}$ after treatment (bottom spectrum B). Based on Figure 1, the distribution of saturated constituents in the range 22-35 in the spectral range is well represented. Not only there are normal alkanes in this range of carbon, but also there are biomarker combinations such as esterans and hopanes. In the ordinary GC, normal alkanes are clearly indicated as the peaks with low density in spectrum " $\mathrm{A}$ " and as compounds in the two-dimensional spectrum. These compounds 
are shown in the 2-dimensional spectrum..$^{19}$ The main sample is seen as blue spots with central red and yellow parts (specimen before the treatment in spectrum "B" in Figure 1A shows the spectrum obtained from the typical GC analysis on the crude oil sample at the lower part of spectrum "B", and the result of the GC X GC, which is treated by the bacteria, and also range of hopanes biomarkers and homologous hopanes with high density at the top of the chromatogram ( $\mathrm{Y}$ axis). ${ }^{20}$ The comparison of the obtained chromatograms (analysis with conventional gas chromatographs and two-dimensional technique) on crude oil samples (before and after the bacterial effect) indicates that Enterobacter aerogenes has been able to have effects on the saturated compounds, especially alkanes; it acts as a suitable metabolism and the saturation compounds with higher carbon can be decomposed slowly. ${ }^{21}$ Reducing the concentration of normal alkanes (typical spectrum as shown in the bottom of Figure 1, in the sample after the treatment) indicates the required effect of the bacteria. ${ }^{22}$ Increasing the concentration of hopane biomarkers in the sample after treatment also shows that the bacterial function on alkane is selectively and consequently after eliminating or reducing the relative alkanes, which appear as red stars at the bottom of the spectrum "B" of Figure 2. A saturated circular structure (hopane biomarkers) ${ }^{23}$ with relatively high concentration in Y-axis is shown in the chromatogram. According to the relative reduction of asphaltene content (Figure 5), regarding the specimen after the treatment, it can be noted that this process causes this bacterium to have effects on the asphaltene and the short-chain structures around the asphaltene micelles section is separated for this reason. $^{24}$
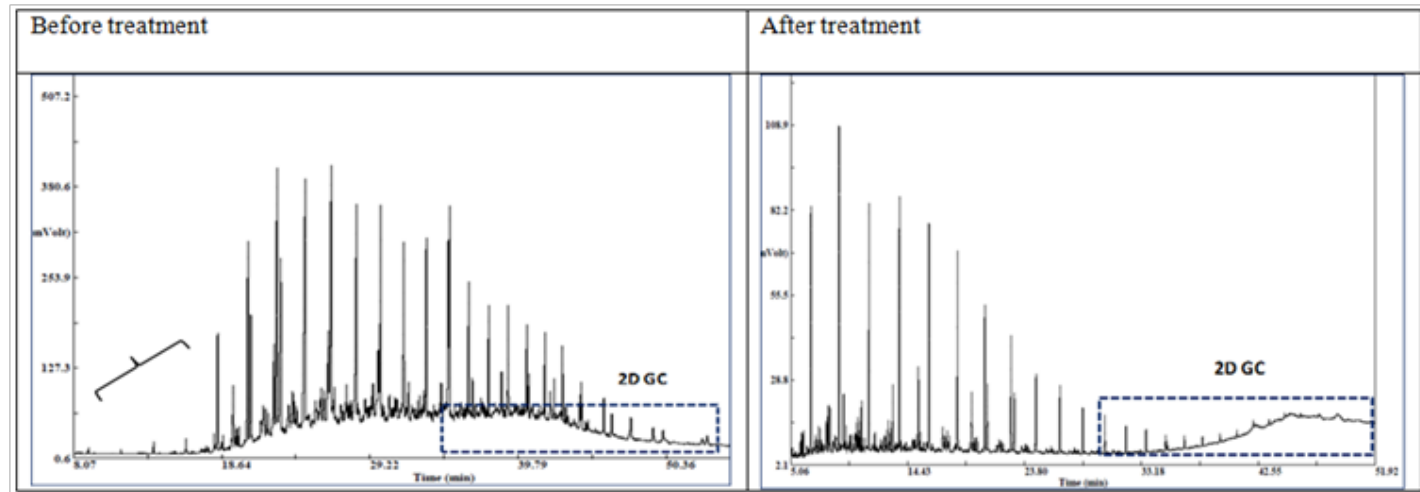

Figure 5 GC test result.

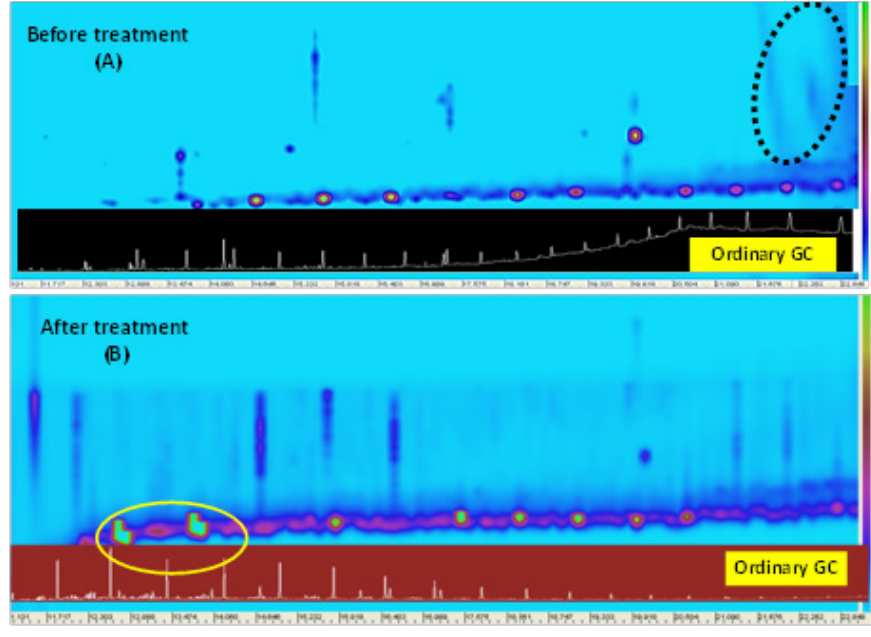

Figure 6 Comparison of conventional and two-dimensional GC spectra obtained from the GC $\times$ GC technique on saturation part from crude oil, before treatment (above spectrum A), after treatment (bottom spectrum B).

Table I SARA test result

\begin{tabular}{lll}
\hline & Before treatment & After treatment \\
\hline Saturate (\%) & 37 & 31 \\
Aromatic (\%) & 35 & 41 \\
Resin (\%) & 17 & 19 \\
Asphalthene (\%) & 11 & 9 \\
\hline
\end{tabular}

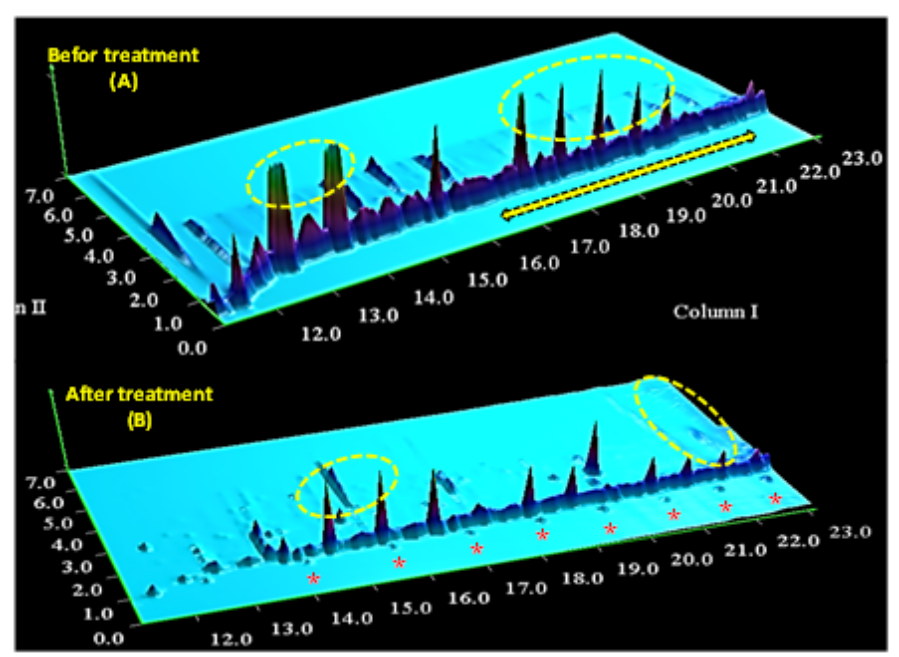

Figure 7 Comparison of two-dimensional spectra obtained from GC $x$ GC technique on crude oil saturation part. (Before treatment: Spectrum A and after treatment: Spectrum B). The scope shown in the two-dimensional analysis is from $\mathrm{C} 22$ to $\mathrm{C} 35$.

The treated sample at the beginning of the chromatogram shows that it has increased the components of light alkenes. Along with this process, the release of hopanes connected to asphaltene and resin has also occurred. Comparison of two-dimensional spectra (see above) $)^{25}$ obtained from GC x GC technique on crude oil saturation (Before: Spectrum "A" and after the treatment: Spectrum "B"). ${ }^{26}$ The scope shown in the two-dimensional analysis is from $\mathrm{C} 22$ to $\mathrm{C} 35$. The effect of enterobacter aerogenes on the crude oil has been studied for the first time, using IR, GC and GCxGC techniques. ${ }^{27}$ This study indicates 
that instead of using carbonic material from amino acid groups in human body the Enterobacter aerogenes applied the required materials from a different part of crude oil. With the IR technique, has been proven that the bacteria in various oil parts uses the structure of oxygen molecules, sulfur, nitrogen and hydrocarbons with weak bonds. ${ }^{28-30}$ In the saturation section, the removal of structures contains hydroxyl $(\mathrm{O}-\mathrm{H})$, nitrogen $(\mathrm{N}-\mathrm{H})$, carbon-carbon, and oxygensulfur structures, including sulfoxide structures. In the aromatic part, $\mathrm{C}=\mathrm{O}$ bounds have been eliminated like in cotton and carbonyl materials. ${ }^{31-33}$ On the other hand, in the resin section, we are faced with the increasing concentration of phenolic, alcoholic and amine compounds, which leads to destruction of hydrocarbon structure in the asphaltene section. Eventually, in the asphaltene section, all the hydrogen, nitrogen, alcoholic structures, and aromatic compounds with a carbon-hydrogen structure are also eliminated. ${ }^{34,35}$ According to SARA results, the highest bacterial effect is on the saturated and asphaltene parts, which are respectively decreased by $6 \%$ and $2 \%$. On the other hand, an increase of $6 \%$ and $2 \%$ occurs in aromatic and resin parts, respectively. ${ }^{36,37}$ Also, in the oil saturated section, and according to the required analysis for $\mathrm{GC}$ and $\mathrm{GCxGC}$ techniques, this bacterium has been able to produce short-chain carbons in the saturated section by destroying the hydrocarbon structures from the asphaltene segment to separate hydrogen, nitrogen, and oxygen. It is worth mentioning that enterobacter aerogenes bacterial releases $\mathrm{CH}_{4}$, which can be used again if the gas is collected.

\section{Acknowledgments}

None.

\section{Conflicts of interest}

The author declares that there are no conflicts of interest.

\section{References}

1. Alan Scragg. Environmental biotechnology. Essex: Longman. University of the West England. 1999

2. Pelletier E, Delille D, Delille B. Crude oil bioremediation in subAntarctic intertidal sediments: chemistry and toxicity of oiled residues. Mar Environ Res. 2004;57(4):311-327.

3. Kosuke Yoshida, Hiroshi Ishii, Yoshimi Ishihara, et al. Bioremediation Potential of Formaldehyde by the Marine Microalga Nannochloropsis oculata ST-3 Strain. Humana Press Journal. 2009;157(2):321-328.

4. Vidali M. Bioremediation. An overview. Pure and Applied Chemistry. 2001;73(7):1163-1172.

5. Sanders WE, Sanders CC. Enterobacter spp.: pathogens poised to flourish at the turn of the century. Clin Microbiol Rev. 1997;10(2):220241.

6. Asadi Nooshin, Zilouei Hamid. Optimization of organosolv pretreatment of rice straw for enhanced biohydrogen production using Enterobacter aerogenes. Bioresource Technology. 2017;227:335-344.

7. Samuel Baron. Medical Microbiology $4^{\text {th }}$ edition. University of Texas Medical Branch at Galveston. 1996.

8. Tianguang Fan, Jianxin Wang, Jill S Buckley. Evaluating Crude Oils by SARA Analysis. Society of Petroleum Engineers. 2002.

9. Robert L Grob, Eugene F Barry. Modern practice of gas chromatography $4^{\text {th }}$ edition. 2004

10. Luigi Mondello. GCxGC Handbook Fundamental principles of comprehensive $2 D$ GC. 2012.
11. Brian C Smith. Fundamentals of Fourier transform infrared spectroscopy, $2^{\text {nd }}$ edition. CRC press. 2011.

12. Pavia DL, Lampman GM, Kriz GS, et al. Introduction to spectroscopy. 2008 .

13. De Champs C, Henquell C, Guelon D, et al. Clinical and bacteriological study of nosocomial infections due to Enterobacter aerogenes resistant to imipenem. Journal of clinical microbiology. 1993;31(1):123-127.

14. Wang JX, Buckley JS. A two-component solubility model of the onset of asphaltene flocculation in crude oils. Energy \& Fuels. 2001;15(5):1004-1012.

15. Updegraff DM, Wren GB. The release of oil from petroleumbearing materials by sulfate-reducing bacteria. Appl Microbiol. 1954;2(6):309-322.

16. Erney DR, Gillespie AM, Gilvydis DM, et al. Explanation of the matrixinduced chromatographic response enhancement of organophosphorus pesticides during open tubular column gas chromatography with splitless or hot on-column injection and flame photometric detection. Journal of Chromatography A. 1993;638(1):57-63.

17. Pinakoulaki E, Soulimane T, Varotsis C. Fourier Transform Infrared (FTIR) and Step-scan Time-resolved FTIR Spectroscopies Reveal a Unique Active Site in Cytochromecaa 3 Oxidase from Thermus thermophilus. J of Biol Chem. 2002;277(36):32867-32874.

18. Silverstein RM, Webster FX, Kiemle DJ, et al. Spectrometric identification of organic compounds. 2007.

19. Marriott P, Shellie R. Principles and applications of comprehensive two-dimensional gas chromatography. TrAC Trends in Analytical Chemistry. 2002;21(9):573-583.

20. Jacobsen NE. NMR spectroscopy explained: simplified theory, applications and examples for organic chemistry and structural biology. 2007.

21. Gillespie S, Hawkey PM. Principles and practice of clinical bacteriology. 2006.

22. Mac Kenzie WR, Hoxie NJ, Proctor ME, et al. A massive outbreak in Milwaukee of Cryptosporidium infection transmitted through the public water supply. New England journal of medicine (NEJM). 1994;331(3):161-167.

23. John T Moore, Richard H Langley. Biochemistry for dummies. 2008.

24. Beens J, Dallüge J, Adahchour M, et al. Moving cryogenic modulator for the comprehensive two-dimensional gas chromatography $(\mathrm{GC} \times \mathrm{GC})$ of surface water contaminants. Journal of Microcolumn Separations. 2001;13(3):134-140.

25. Derrick MR, Stulik D, Landry JM. Infrared spectroscopy in conservation science. Getty Publications. 2000.

26. Field LD, Sternhell S, Kalman JR. Organic structures from spectra. 2012.

27. PellizzariED, HartwellTD, Harris BS, etal. Purgeable organic compounds in mother's milk. Bull Environ Contam Toxicol. 1982;28(3):322-328.

28. Stuart BH. Organic molecules. Infrared spectroscopy: Fundamentals and applications. 2004;71-93

29. Griffiths PR, De Haseth JA. Fourier transform infrared spectrometry. John Wiley \& Sons publications. 2007.

30. Mayo DW, Miller FA, Hannah RW. Course notes on the interpretation of infrared and Raman spectra. John Wiley \& Sons Inc. 2004.

31. Grob RL, Barry EF. Modern practice of gas chromatography. John Wiley \& Sons, Inc. 2004. 
32. Stosur JG, Slater JA. Recovery Methods for Heavy Oil and Tar Sands: Section VI. 1987.

33. Dhail S. Microbial enhanced oil recovery using potent biosurfactant produced by Pseudomonas sp. from Arabian Sea, Mumbai. Journal of petroleum and gas engineering. 2013;4(3):57-60.

34. Donaldson EC, Chilingarian GV, Yen TF. Microbial enhanced oil recovery. 1989

35. Donaldson EC. Microbial enhancement of oil recovery-recent advances, volume 39. Elsevier. 1991.
36. Banat IM. Biosurfactants production and possible uses in microbial enhanced oil recovery and oil pollution remediation: a review. Bioresource technology. 1995;51(1):1-12.

37. Shah A, Fishwick R, Wood J, et al. A review of novel techniques for heavy oil and bitumen extraction and upgrading. Energy \& Environmental Science. 2010;3(6):700-714. 